\title{
Altered spontaneous brain activity pattern in patients with high myopia using amplitude of low- frequency fluctuation: a resting-state fMRI study
}

This article was published in the following Dove Press journal:

Neuropsychiatric Disease and Treatment

14 November 2016

Number of times this article has been viewed

\author{
Xin Huang ${ }^{1,2, *}$ \\ Fu-Qing Zhou ${ }^{3, *}$ \\ Yu-Xiang $\mathrm{Hu}^{\prime}$ \\ Xiao-Xuan Xu' \\ Xiong Zhou ${ }^{4}$ \\ Yu-Lin Zhong \\ Jun Wang ${ }^{4}$ \\ Xiao-Rong $\mathrm{Wu}^{\prime}$
}

'Department of Ophthalmology, The First Affiliated Hospital of Nanchang University, Nanchang, ${ }^{2}$ Department of Ophthalmology, The First People's Hospital of Jiujiang City, Jiujiang, ${ }^{3}$ Department of Radiology, The First Affiliated Hospital of Nanchang University, Jiangxi Province Medical Imaging Research Institute, ${ }^{4}$ Second Department of Respiratory Disease, Jiangxi Provincial People's Hospital, Nanchang, Jiangxi, People's Republic of China

*These authors contributed equally to this work

Correspondence: Xiao-Rong Wu Department of Ophthalmology, The First Affiliated Hospital of Nanchang University, 17 YongWai Zheng Street, DongHu District, Nanchang 330006, Jiangxi, People's Republic of China

Tel +86 I36 I7093259

Email wxr9802।@|26.com

Jun Wang

Second Department of Respiratory Disease, Jiangxi Provincial People's Hospital, 152 Aiguo Road, DongHu District, Nanchang 330006, Jiangxi,

People's Republic of China

Tel +86 I897005 5686

Email wangjun5087@163.com
Objective: Many previous reports have demonstrated significant neural anatomy changes in the brain of high myopic (HM) patients, whereas the spontaneous brain activity changes in the HM patients at rest are not well studied. Our objective was to use amplitude of low-frequency fluctuation (ALFF) method to investigate the changes in spontaneous brain activity in HM patients and their relationships with clinical features.

Methods: A total of 38 patients with HM (17 males and 21 females) and 38 healthy controls (HCs) (17 males and 21 females) closely matched in age, sex, and education underwent restingstate functional magnetic resonance imaging scans. The ALFF method was used to assess local features of spontaneous brain activity. The relationship between the mean ALFF signal values in many brain regions and the clinical features in HM patients was calculated by correlation analysis.

Results: Compared with HCs, the HM patients had significantly lower ALFF in the right inferior and middle temporal gyrus, left middle temporal gyrus, left inferior frontal gyrus/putamen, right inferior frontal gyrus/putamen/insula, right middle frontal gyrus, and right inferior parietal lobule and higher ALFF values in the bilateral midcingulate cortex, left postcentral gyrus, and left precuneus/inferior parietal lobule. However, no relationship was found between the mean ALFF signal values of the different areas and the clinical manifestations in HM.

Conclusion: The HM patients were affected with brain dysfunction in many regions, which may indicate the presence of neurobiological changes involving deficits in language understanding and attentional control in HM patients.

Keywords: ALFF, high myopic, resting state, functional magnetic resonance imaging

\section{Introduction}

High myopia (HM) is a common eye disorder which has become a public health problem globally. ${ }^{1}$ The prevalence of myopia is $70 \%$ among teenagers in Hong Kong. ${ }^{2}$ The occurrence of HM is associated with many factors such as genetic variants, ${ }^{3}$ education factors, ${ }^{4}$ and season of birth. ${ }^{5}$ Clinically, $\mathrm{HM}$ is often associated with low best-corrected visual acuity (VA). Besides, HM may lead to macular complications, ${ }^{6}$ chorioretinal atrophy, ${ }^{7}$ and retinal detachment. ${ }^{8}$ Currently, the important methods for HM correction include among other excimer laser ${ }^{9}$ and orthokeratology. ${ }^{10}$

Optical coherence tomography (OCT) is a noninvasive, high-resolution method that can measure the structure of the eye accurately. A previous study demonstrated using OCT method that the HM eyes have less retinal nerve fiber thickness compared with emmetropic eyes. ${ }^{11}$ Another study reported that the OCT examinations could reveal the types of abnormalities around the optic disc in HM eyes. ${ }^{12}$ Meanwhile, OCT 
can evaluate HM-related complications such as choroidal neovascularization. ${ }^{13}$ High-resolution magnetic resonance (MR) also has been used to evaluate the eye shape in HM. MR could make correct assessment of the shape of eye in HM patients. ${ }^{14}$ The aforementioned inspection methods focus merely on the pathological changes of ocular trauma in HM. However, other visual systems, including the visual pathways and the visual cortex, are often overlooked.

Functional magnetic resonance imaging (fMRI) has been used to evaluate the brain functional connectivity in HM patients. Zhai et al reported that HM patients showed decreased short- and long-range functional connectivity density in the posterior cingulate cortex/precuneus. ${ }^{15}$ However, there are only a few studies on the changes of spontaneous brain activity in HM patients at rest. The blood oxygenation level dependent signal changes can reflect the changes in the neuronal and physiological activities to some extent. Amplitude of low-frequency fluctuation (ALFF) can be used to detect the spontaneous neuronal activity in blood oxygenation level dependent signal. The ALFF is a reliable relevant amplitude measure which can be detected in the human resting brain. ${ }^{16}$ The ALFF has been widely applied to detect the brain function within a specific frequency range $(0.01-0.08 \mathrm{~Hz}) .{ }^{17}$ In our previous studies, the ALFF method was successfully used to assess the neurological conditions in some eye diseases such as optic neuritis, ${ }^{18}$ glaucoma, ${ }^{19}$ and comitant strabismus. ${ }^{20}$ Our study is the first to use the ALFF method to investigate the spontaneous brain activity in HM patients and investigate its relationship with the behavioral performances.

\section{Materials and methods Subjects}

A total of 38 patients with HM (17 males and 21 females) were recruited from the ophthalmology department of the First Affiliated Hospital of Nanchang University. The inclusion criteria of the study were: 1) HM patients with binocular refractive errors; 2) binocular refractive diopter -6.00 to $-7.00 \mathrm{D}$ and corrected VA >1.0; and 3) absence of ocular diseases (cataracts, glaucoma, optic neuritis, retinal degeneration) in both eyes. The exclusion criteria were: 1) refractive diopter -1.00 to $-6.00 \mathrm{D} ; 2$ ) monocular HM; 3) congenital HM; 4) HM with amblyopia and related complications (retinal detachment, retinal atrophy degeneration, macular hole); 5) long-term medical treatment of HM; and 6) psychiatric disorders and cerebral infarction diseases (cerebral hemorrhage, cerebral infarction, cerebral vascular malformations).

Thirty-eight healthy controls (HCs; 17 males, 21 females) who were matched in age, sex, and educational status to subjects in the HM group were also recruited for this study. All healthy subjects met the following criteria: 1) no ocular disease with uncorrected or corrected $\mathrm{VA}>1.0$; 2) no psychiatric disorders (depression, bipolar disorder, sleep disorder); and 3) could be scanned with MRI (eg, no cardiac pacemaker or implanted metal devices). All research methods followed the Declaration of Helsinki and the study protocol was approved by the ethics committee of the First Affiliated Hospital of Nanchang University. All volunteers participated voluntarily and were informed of the purposes, methods, and potential risks before they signed an informed consent form.

\section{MRI parameters}

MRI scanning was performed on a 3 T MR scanner (Trio; Siemens, Munich, Germany). The functional data were obtained with spoiled gradient-recalled echo sequence with the parameters (repetition time $=1,900 \mathrm{~ms}$, echo time $=2.26 \mathrm{~ms}$, thickness $=1.0 \mathrm{~mm}$, gap $=0.5 \mathrm{~mm}$, acquisition matrix $=256 \times 256$, field of view $=250 \times 250 \mathrm{~mm}$, flip angle $=9^{\circ}$ ). We also obtained 240 functional images (repetition time $=2,000 \mathrm{~ms}$, echo time $=30 \mathrm{~ms}$, thickness $=4.0 \mathrm{~mm}$, gap $=1.2 \mathrm{~mm}$, acquisition matrix $=64 \times 64$, flip angle $=90^{\circ}$, field of view $=220 \times 220 \mathrm{~mm}, 29$ axial).

\section{fMRI data analysis}

Functional data were classified by MRIcro software (www.MRIcro.com) and incomplete data were removed. The first ten volumes were discarded due to magnetization equilibration. The rest of the data was preprocessed by Data Processing Asisstant for Resting-State fMRI, version 2.3 (http://rfmri.org/DPARSF) software, including slice timing, head motion correction, spatial normalization, and smoothing with a Gaussian kernel of $6 \times 6 \times 6 \mathrm{~mm}^{3}$ full width at half maximum. The fMRI images were spatially normalized to the Montreal Neurological Institute space criteria using the standard echo-planar imaging template and resampling the images at a resolution of $3 \times 3 \times 3 \mathrm{~mm}$. After preprocessing, the ALFF of each voxel was divided by the global mean ALFF value for each participant. More details of ALFF calculation have been reported in our previous study. ${ }^{11}$

\section{Statistical analysis}

A one-sample $t$-test was performed to extract the ALFF results across the subjects within each group $(P<0.05)$. Statistical analysis was performed with a general linear model analysis using the Statistical Parametric Mapping 8 (SPM8) toolkit to investigate the ALFF signal group differences in the resting state between the two groups, after controlling 
for the effects of age and sex $(P<0.01$ for multiple comparisons using Gaussian random field theory) $(z>2.3, P<0.01$, cluster $>40$ voxels, AlphaSim corrected).

\section{Brain-behavior correlation analysis}

Brain areas with different ALFF findings between the two groups were classified as regions of interest with the Resting-State fMRI Data Analysis Toolkit version 1.8 software. The relationship between the mean ALFF value in different brain regions in the HM group and the behavioral performances was calculated with correlation analysis $(P<0.05$ was considered statistically significant difference).

\section{Clinical data analysis}

The cumulative clinical measurements, including the duration of the onset of HM, refractive diopter, and best-corrected VA, were recorded and analyzed in the study with independent sample $t$-test $(P<0.05$ was considered statistically significant difference).

\section{Results \\ Demographics and visual measurements}

There were no marked differences in weight $(P=0.972)$, age $(P=0.856)$, best-corrected VA-right $(P=0.705)$, and best-corrected VA-left $(P=0.940)$ between the two groups (Table 1).

\section{ALFF differences}

A one-sample $t$-test was performed to extract the ALFF results across the subjects within each group $(P<0.05)$. Intragroup comparison within the $\mathrm{HM}$ and $\mathrm{HC}$ groups is shown in Figure 1. Compared with HCs, HMs had significantly lower ALFF in the right inferior and middle temporal gyrus (MTG), left MTG, left inferior frontal gyrus (IFG)/ putamen, right IFG/putamen/insula, right middle frontal gyrus, and right inferior parietal lobule (IPL) (Figure 2, blue areas; Table 2). In contrast, higher ALFF values were observed in the bilateral midcingulate cortex (MCC), left postcentral gyrus, and left precuneus/IPL in the HM group (Figure 2, red areas; Table 2). The mean values of altered ALFF between the two groups are shown in Figure 3. In the HM group, there was no significant correlation between the mean ALFF values in different brain regions and the clinical manifestations $(P>0.05)$.

\section{Receiver operating characteristic (ROC) curve}

We hypothesized that the ALFF differences between the HM and $\mathrm{HC}$ groups might be useful diagnostic markers. Besides, the mean ALFF values in different brain regions were analyzed by ROC curve method. The areas under the ROC curve for the ALFF values were as follows: right inferior and MTG (0.744), left MTG (0.734), left IFG/putamen (0.713), right $\mathrm{IFG} /$ putamen/insula $(0.725)$, right middle frontal gyrus (0.783), right IPL (0.729) (HMs < HCs) (Figure 4A); bilateral MCC (0.643), left postcentral gyrus (0.679), left precuneus/IPL (0.659) (HMs $>$ HCs) (Figure 4B).

\section{Discussion}

In our study, the HM patients had significantly lower ALFF in the right inferior and MTG, left MTG, left IFG/putamen, right IFG/putamen/insula, right middle frontal gyrus, and right IPL and higher ALFF values in the bilateral MCC, left postcentral gyrus, and left precuneus/IPL.

\section{Analysis of the decreased ALFF values in $\mathrm{HM}$}

The IFG is a part of the frontal lobe which plays an important role in language comprehension. ${ }^{21}$ A previous study showed that the left IFG is a critical brain area in reading the mind in the eyes. ${ }^{22}$ The triangular part of the IFG is the grammar center. ${ }^{23}$

Table I Demographics and clinical measurements by group

\begin{tabular}{|c|c|c|c|c|}
\hline Variable & HM & HC & t-test & P-value* \\
\hline Male/female, $n$ & $|7 / 2|$ & $|7 / 2|$ & $\mathrm{N} / \mathrm{A}$ & $>0.99$ \\
\hline Age (years), mean $\pm \mathrm{SD}$ & $22.76 \pm 1.28$ & $22.7 I \pm I .23$ & 0.183 & 0.856 \\
\hline Weight $(\mathrm{kg})$, mean \pm SD & $65.21 \pm 6.89$ & $65.26 \pm 6.31$ & -0.035 & 0.972 \\
\hline Handedness, $n$ & $38 \mathrm{R}$ & $38 \mathrm{R}$ & $\mathrm{N} / \mathrm{A}$ & $>0.99$ \\
\hline Duration of HM (years), mean \pm SD & $6.34 \pm 1.12$ & N/A & $\mathrm{N} / \mathrm{A}$ & N/A \\
\hline Refractive diopter-right (D), mean \pm SD & $-641.05 \pm 37.04$ & N/A & $\mathrm{N} / \mathrm{A}$ & N/A \\
\hline Refractive diopter-left (D), mean \pm SD & $-643.68 \pm 37.59$ & $\mathrm{~N} / \mathrm{A}$ & N/A & N/A \\
\hline Best-corrected VA-right eye, mean \pm SD & $1.15 \pm 0.16$ & $1.13 \pm 0.15$ & 0.381 & 0.705 \\
\hline Best-corrected VA-left eye, mean \pm SD & $1.14 \pm 0.14$ & $1.14 \pm 0.16$ & -0.076 & 0.940 \\
\hline
\end{tabular}

Note: $* P<0.05$, independent $t$-tests for comparing two groups.

Abbreviations: HC, healthy control; HM, high myopia; N/A, not applicable; R, right; VA, visual acuity. 

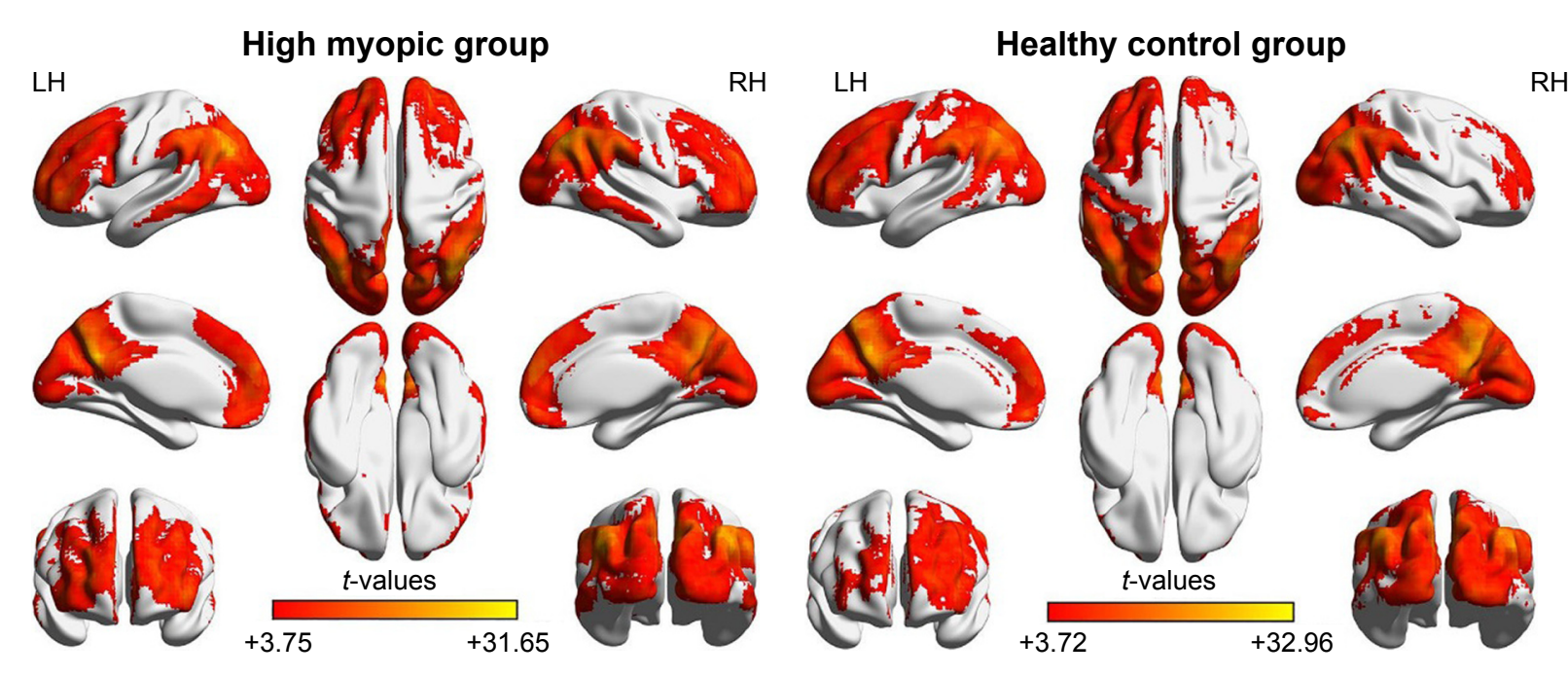

Healthy control group

Figure I One-sample $t$-test results. Within-group ALFF maps within the HM (left) and HC (right) groups $(P<0.00$ I, FDR corrected).

Abbreviations: ALFF, amplitude of low-frequency fluctuation; FDR, false discovery rate; HC, healthy control; HM, high myopia; LH, left hemisphere; RH, right hemisphere.

Besides, another study reported that there were changes in morphology of the IFG in language-disordered subjects. ${ }^{24}$ Meanwhile, the right IFG plays a vital role in inhibition and attentional control. ${ }^{25} \mathrm{~A}$ previous research showed that stimulation of the right IFG may improve attention in patients with early Alzheimer's disease. ${ }^{26} \mathrm{Li}$ et al demonstrated that patients with HM showed smaller white matter volume in the prefrontal area, compared with HCs. ${ }^{27}$ In support of these findings, we observed that HM patients had significantly lower ALFF in the bilateral IFG. We surmised that HM may lead to dysfunction of the bilateral IFG, which might indicate that HM patients suffer from impairment of language understanding and attentional control.

The putamen is located in the forebrain, which connects to the substantia nigra and globus pallidus. The putamen is a part of the dorsal striatum. It is also one of the structures that comprise the basal ganglia. A previous study demonstrated that the putamen was involved in processing the motor information in monkeys. ${ }^{28}$ Another study reported that macaque monkey showed increased activity of neurons in the putamen during a visuomotor task. ${ }^{29}$ Apart from its involvement in motor skills, the putamen also plays an important role in learning. ${ }^{30-32}$ In addition, dysfunction of the putamen was associated with many diseases such as schizophrenia, ${ }^{33}$ pain, ${ }^{34}$ and Parkinson disease. ${ }^{35}$ In our study, we found that HM patients had significantly lower ALFF in the bilateral putamen. We speculated that HM may lead to dysfunction of the putamen, which may be reflected as defective movement and learning in HM patients.

MTG is located in the middle of superior temporal gyrus and inferior temporal gyrus, and is involved in speed of word recognition. ${ }^{36}$ Besides, the MTG includes middle temporal

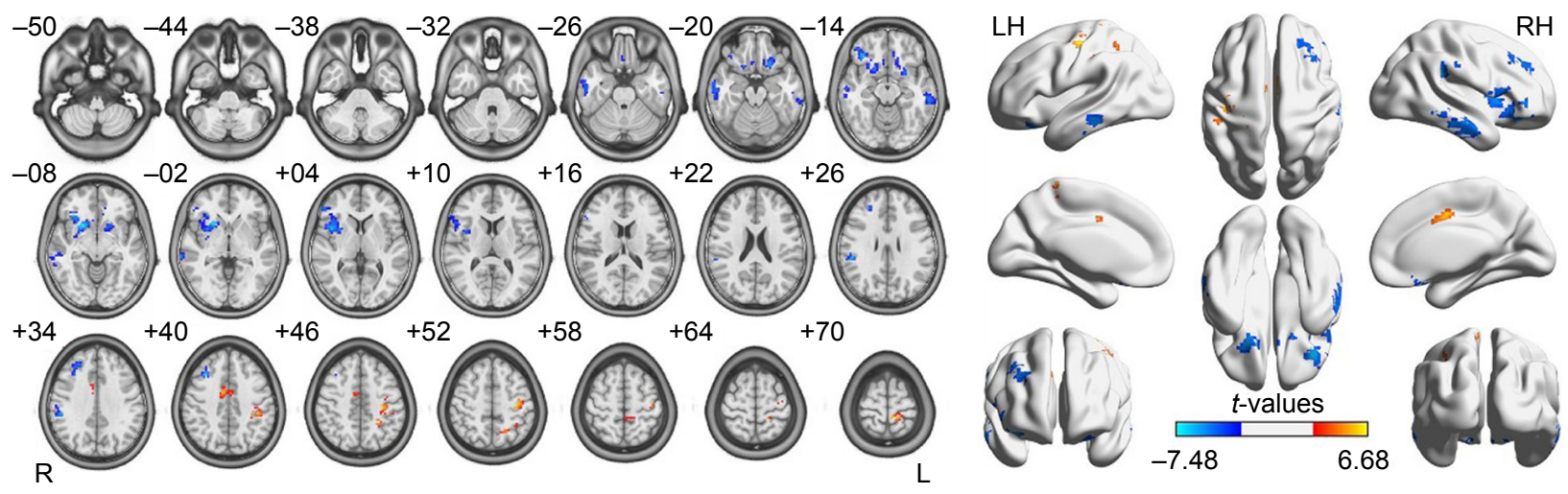

Figure 2 Spontaneous brain activity in the monocular blindness and healthy control groups.

Notes: Significant differences in activity were observed in the right inferior and middle temporal gyrus, left middle temporal gyrus, left inferior frontal gyrus/putamen, right inferior frontal gyrus/putamen/insula, right middle frontal gyrus, right inferior parietal lobule, bilateral midcingulate cortex, left postcentral gyrus, and left precuneus/inferior parietal lobule. The red or yellow areas denote higher ALFF values and the blue areas indicate lower ALFF values $(P<0.0$ I for multiple comparisons using GRF theory) ( $z>2.3$, $P<0.0$ I, cluster $>40$ voxels, AlphaSim corrected).

Abbreviations: ALFF, amplitude of low-frequency fluctuation; GRF, Gaussian random field; L, left; LH, left hemisphere; R, right; RH, right hemisphere. 
Table 2 Brain areas with significantly different ALFF values between the groups

\begin{tabular}{|c|c|c|c|c|c|c|c|}
\hline \multirow[t]{2}{*}{ Brain areas } & \multicolumn{3}{|c|}{ MNI coordinates } & \multirow[t]{2}{*}{ Voxels } & \multirow[t]{2}{*}{ BA } & \multirow[t]{2}{*}{$\mathbf{L} / \mathbf{R}$} & \multirow{2}{*}{$\begin{array}{l}\text { Peak } \\
\text { t-values }\end{array}$} \\
\hline & $x$ & $y$ & $\mathbf{z}$ & & & & \\
\hline \multicolumn{8}{|l|}{$\mathrm{HM}<\mathrm{HC}$} \\
\hline Inferior and middle temporal gyrus & 60 & -27 & -18 & 147 & 20.21 & $\mathrm{R}$ & -5.323 \\
\hline Middle temporal gyrus & -63 & -33 & -15 & 68 & 21 & $\mathrm{~L}$ & -5.681 \\
\hline Inferior frontal gyrus/putamen & -21 & 24 & -21 & 119 & 47 & L & -5.975 \\
\hline Inferior frontal gyrus/putamen/insula & 21 & 15 & -6 & 450 & 47.13 & $\mathrm{R}$ & -7.484 \\
\hline Middle frontal gyrus & 33 & 24 & 39 & 86 & 9 & $\mathrm{R}$ & -6.986 \\
\hline Inferior parietal lobule & 51 & -33 & 30 & 60 & 40 & $\mathrm{R}$ & -6.631 \\
\hline \multicolumn{8}{|l|}{$\mathrm{HM}>\mathrm{HC}$} \\
\hline Midcingulate cortex & 9 & -3 & 39 & 59 & 24 & B & 5.874 \\
\hline Postcentral gyrus & -39 & -15 & 54 & 159 & 3 & $\mathrm{~L}$ & 6.679 \\
\hline Precuneus/inferior parietal lobule & -27 & -48 & 48 & 37 & 40.7 & L & 6.479 \\
\hline
\end{tabular}

Note: The statistical threshold was set at the voxel level with $P<0.05$ for multiple comparisons using GRF theory ( $>2.3, P<0.0$ l, cluster $>40$ voxels, AlphaSim corrected).

Abbreviations: ALFF, amplitude of low-frequency fluctuation; B, bilateral; BA, Brodmann area; GRF, Gaussian random field; HC, healthy control; HM, high myopia; L, left; MNI, Montreal Neurological Institute; R, right.

visual area (V5), which plays an important role in visual motion. ${ }^{37,38}$ In addition, the MTG is responsible for the pursuit eye movements..$^{39}$ In our study, we found that HM patients had significantly decreased ALFF values in the bilateral MTG, indicating the impairment of the MTG. We speculated that HM may be associated with deficits in visual motion and word recognition.

\section{Analysis of the increased ALFF values in HM}

The IPL is located behind the lower part of the postcentral sulcus. It is involved in the encoding of spatial location. ${ }^{40}$ Besides, the IPL is responsible for the spatial attention or motor attention..$^{41}$ Meanwhile, the IPL is involved in the auditory motion. ${ }^{42}$ In our study, we found that HM patients had significantly higher ALFF values in the left IPL, which indicated increased activities in the left IPL in them. We speculated that the higher ALFF in the left IPL may indicate reinforcement of the spatial attention in HM patients.

The cingulate cortex is located in the middle of the cerebral cortex, which is a part of limbic cortex. The MCC plays an important role in cognitive control. ${ }^{43}$ Besides, the MCC may be involved in negative emotion and motor control. ${ }^{44,45}$ Dysfunction of the MCC leads to many diseases such as pain $^{46}$ and autism. ${ }^{47}$ In our study, we found that there were

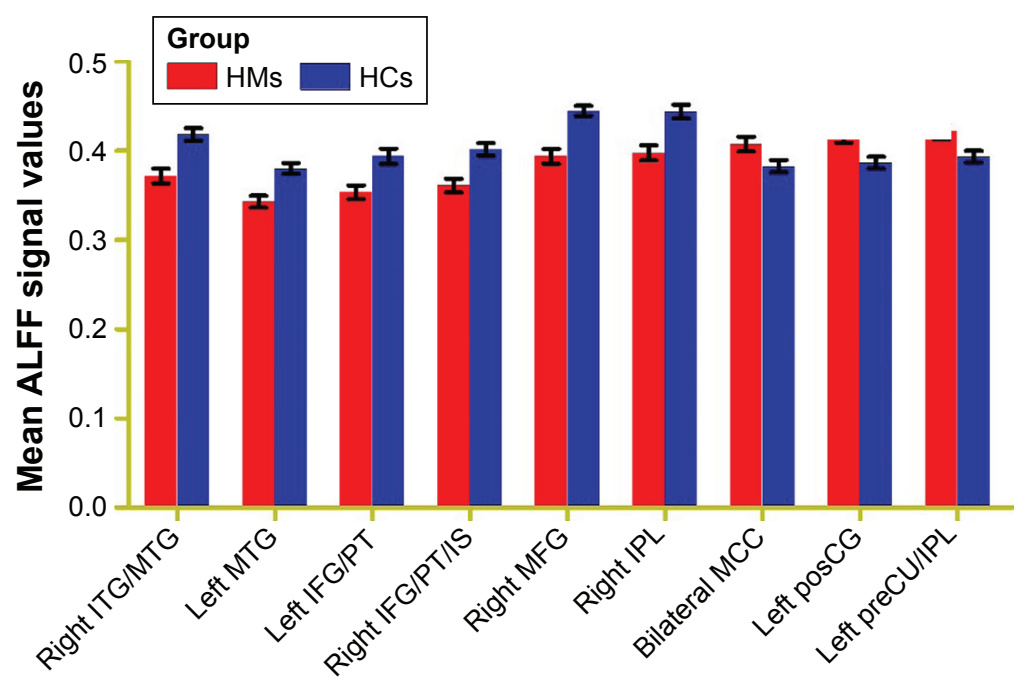

\section{Altered ALFF regions}

Figure 3 The mean values of altered ALFF values between the $H M$ and $H C$ groups.

Abbreviations: ALFF, amplitude of low-frequency fluctuation; HC, healthy control; HM, high myopia; IFG, inferior frontal gyrus; IPL, inferior parietal lobule; IS, insula; ITG, inferior temporal gyrus; MCC, midcingulate cortex; MFG, middle frontal gyrus; MTG, middle temporal gyrus; posCG, postcentral gyrus; preCU, precuneus; PT, putamen. 

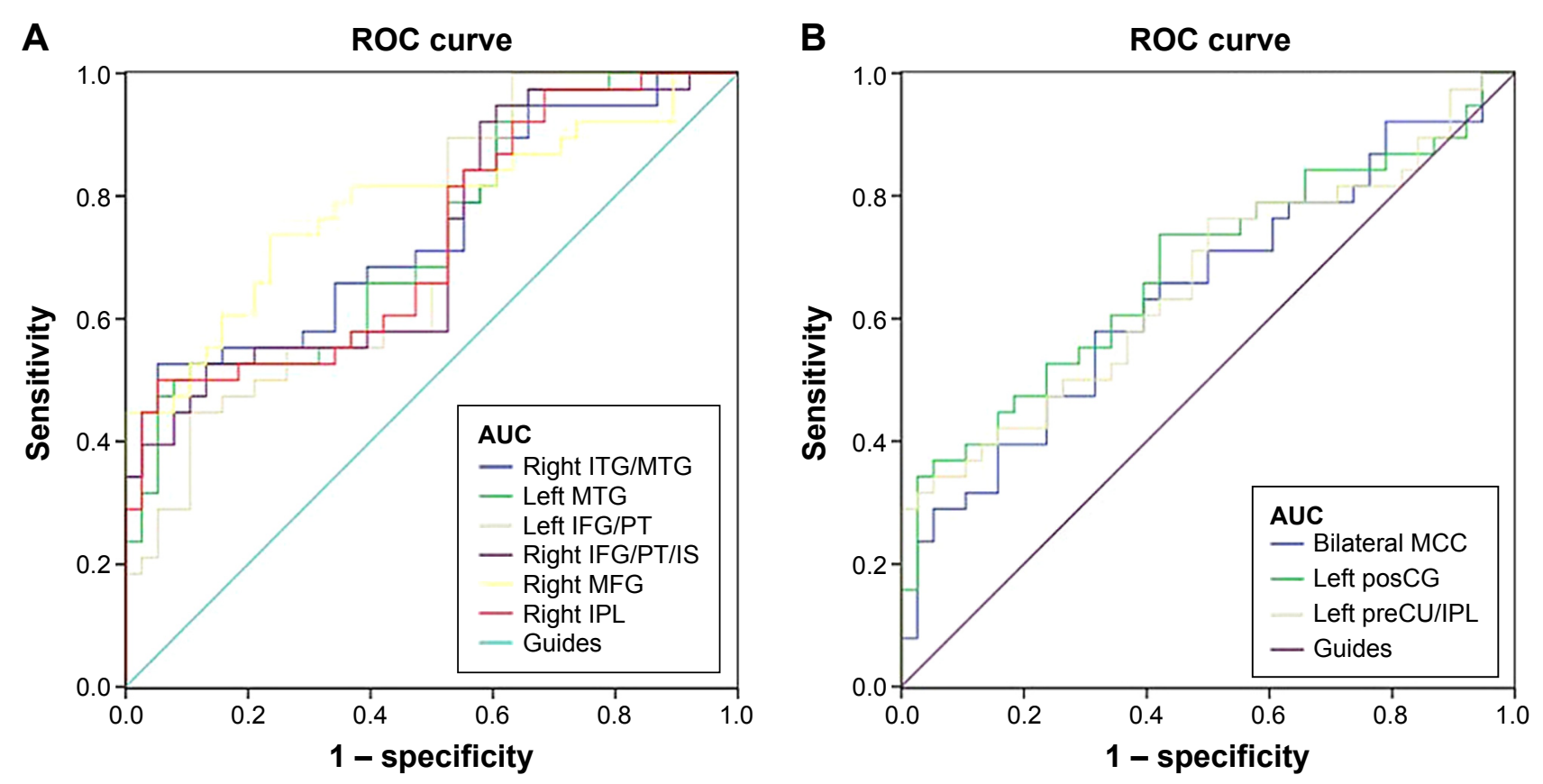

Figure 4 ROC curve analysis of the mean ALFF values for altered brain regions.

Notes: The AUCs were: $0.744(P<0.00$ I; $95 \% \mathrm{Cl}$ : $0.634-0.855)$ for the right ITG/MTG, $0.734(P<0.00$ I; $95 \%$ Cl: $0.634-0.845)$ for the left MTG, 0.7 I3 ( $P=0.00$ I; 95\% Cl: $0.598-0.828)$ for the left IFG/PT, 0.725 ( $P=0.00 \mathrm{I}$; $95 \% \mathrm{Cl}$ : $0.6 \mathrm{II}-0.839)$ for the right IFG/PT/IS, 0.783 ( $P<0.00 \mathrm{I}$; $95 \% \mathrm{Cl}$ : $0.677-0.888)$ for the right MFG, and 0.729 ( $P=0.00$ I; $95 \%$ Cl: $0.616-0.84 I)$ for the right IPL (HMs < HCs) (A). The AUCs were: 0.643 ( $P=0.032$; $95 \%$ Cl: $0.519-0.768)$ for the bilateral MCC, 0.679 ( $P=0.007$; 95\% Cl: 0.558-0.80I) for the left posCG, 0.659 ( $P=0.017 ; 95 \% \mathrm{Cl}: 0.536-0.783)$ for the left preCU/IPL (HMs $>\mathrm{HCs})(\mathbf{B})$.

Abbreviations: ALFF, amplitude of low-frequency fluctuation; AUC, area under the curve; $\mathrm{Cl}$, confidence interval; $\mathrm{HCs}$, healthy controls; HM, high myopia; IFG, inferior frontal gyrus; IPL, inferior parietal lobule; IS, insula; ITG, inferior temporal gyrus; MCC, midcingulate cortex; MFG, middle frontal gyrus; MTG, middle temporal gyrus; posCG, postcentral gyrus; preCU, precuneus; PT, putamen; ROC, receiver operating characteristic.

significantly higher ALFF values in the bilateral MCC in HM patients, which indicated enhanced activities in bilateral MCC. We surmised that the HM may lead to the hyperfunction of emotion and attention.

\section{Limitations}

However, there are some limitations in our study, such as the relatively small sample size and the differences in refractive diopter in HM patients. Besides, the duration of the onset of HM is not exactly the same, which may have a bad effect on the accuracy of the results.

\section{Conclusion}

In summary, our results showed that there were abnormal spontaneous activities in many brain regions, which may indicate that neurobiological changes associated with deficits in language understanding and attentional control are found in HM patients.

\section{Acknowledgments}

This was not an industry-supported study. This study was supported by the National Natural Science Foundation of China (81360151 and 81460004), Technology and Science
Foundation of Jiangxi Province (20141BBG70027), Jiangxi Province Education Department Scientific Research Foundation (GJJ13147), and Health Development Planning Commission Science Foundation of Jiangxi Province (20141031).

\section{Disclosure}

The authors report no conflicts of interest in this work.

\section{References}

1. Holden BA, Fricke TR, Wilson DA, et al. Global prevalence of myopia and high myopia and temporal trends from 2000 through 2050. Ophthalmology. 2016;123(5):1036-1042.

2. Edwards MH, Lam CS. The epidemiology of myopia in Hong Kong. Ann Acad Med Singapore. 2004;33(1):34-38.

3. Xie M, Li Y, Wu J, Wu J. Genetic variants in MiR-29a associated with high myopia. Ophthalmic Genet. Epub 2016 Feb 17:1-3.

4. Jonas JB, Xu L, Wang YX, et al. Education-related parameters in high myopia: adults versus school children. PLoS One. 2016;11(5): $\mathrm{e} 0154554$.

5. McMahon G, Zayats T, Chen YP, Prashar A, Williams C, Guggenheim JA. Season of birth, daylight hours at birth, and high myopia. Ophthalmology. 2009;116(3):468-473.

6. Lichtwitz O, Boissonnot M, Mercié M, Ingrand P, Leveziel N. Prevalence of macular complications associated with high myopia by multimodal imaging. J Fr Ophtalmol. 2016;39(4):355-363.

7. Ohno-Matsui K, Jonas JB, Spaide RF. Macular bruch membrane holes in highly myopic patchy chorioretinal atrophy. Am J Ophthalmol. 2016; $166: 22-28$ 
8. Alkabes M, Burés-Jelstrup A, Salinas C, et al. Macular buckling for previously untreated and recurrent retinal detachment due to high myopic macular hole: a 12-month comparative study. Graefes Arch Clin Exp Ophthalmol. 2014;252(4):571-581.

9. Reinstein DZ, Carp GI, Archer TJ, et al. Long-term visual and refractive outcomes after LASIK for high myopia and astigmatism from -8.00 to -14.25 D. J Refract Surg. 2016;32(5):290-297.

10. Charm J, Cho P. High myopia-partial reduction ortho-k: a 2-year randomized study. Optom Vis Sci. 2013;90(6):530-539.

11. Malakar M, Askari SN, AshrafH, Waris A, Ahuja A, Asghar A. Optical coherence tomography assisted retinal nerve fibre layer thickness profile in high myopia. J Clin Diagn Res. 2015;9(2):NC01-NC03.

12. Shimada N, Ohno-Matsui K, Nishimuta A, Tokoro T, Mochizuki M. Peripapillary changes detected by optical coherence tomography in eyes with high myopia. Ophthalmology. 2007;114(11):2070-2076.

13. Baba T, Ohno-Matsui K, Yoshida T, et al. Optical coherence tomography of choroidal neovascularization in high myopia. Acta Ophthalmol Scand. 2002;80(1):82-87.

14. Moriyama M, Ohno-Matsui K, Modegi T, et al. Quantitative analyses of high-resolution 3D MR images of highly myopic eyes to determine their shapes. Invest Ophthalmol Vis Sci. 2012;53(8):4510-4518.

15. Zhai L, Li Q, Wang T, et al. Altered functional connectivity density in high myopia. Behav Brain Res. 2016;303:85-92.

16. Zuo XN, Di Martino A, Kelly C, et al. The oscillating brain: complex and reliable. Neuroimage. 2010;49(2):1432-1445.

17. Zang YF, He Y, Zhu CZ, et al. Altered baseline brain activity in children with ADHD revealed by resting-state functional MRI. Brain Dev. 2007;29(2):83-91.

18. Huang X, Cai FQ, Hu PH, et al. Disturbed spontaneous brainactivity pattern in patients with optic neuritis using amplitude of lowfrequency fluctuation: a functional magnetic resonance imaging study. Neuropsychiatr Dis Treat. 2015;11:3075-3083.

19. Huang X, Zhong YL, Zeng XJ, et al. Disturbed spontaneous brain activity pattern in patients with primary angle-closure glaucoma using amplitude of low-frequency fluctuation: a fMRI study. Neuropsychiatr Dis Treat. 2015;11:1877-1883.

20. Tan G, Huang X, Zhang Y, et al. A functional MRI study of altered spontaneous brain activity pattern in patients with congenital comitant strabismus using amplitude of low-frequency fluctuation. Neuropsychiatr Dis Treat. 2016;12:1243-1250.

21. Manjaly ZM, Marshall JC, Stephan KE, Gurd JM, Zilles K, Fink GR. Context-dependent interactions of left posterior inferior frontal gyrus in a local visual search task unrelated to language. Cogn Neuropsychol. 2005;22(3):292-305.

22. Dal Monte O, Schintu S, Pardini M, et al. The left inferior frontal gyrus is crucial for reading the mind in the eyes: brain lesion evidence. Cortex. 2014;58:9-17.

23. Nauchi A, Sakai KL. Greater leftward lateralization of the inferior frontal gyrus in second language learners with higher syntactic abilities. Hum Brain Mapp. 2009;30(11):3625-3635.

24. Clark MM, Plante E. Morphology of the inferior frontal gyrus in developmentally language-disordered adults. Brain Lang. 1998;61(2): 288-303.

25. Hampshire A, Chamberlain SR, Monti MM, Duncan J, Owen AM. The role of the right inferior frontal gyrus: inhibition and attentional control. Neuroimage. 2010;50(3):1313-1319.

26. Eliasova I, Anderkova L, Marecek R, Rektorova I. Non-invasive brain stimulation of the right inferior frontal gyrus may improve attention in early Alzheimer's disease: a pilot study. J Neurol Sci. 2014;346(1-2): 318-322.

27. Li Q, Guo M, Dong H, Zhang Y, Fu Y, Yin X. Voxel-based analysis of regional gray and white matter concentration in high myopia. Vision Res. 2012;58:45-50.
28. Vicente AF, Bermudez MA, Romero Mdel C, Perez R, Gonzalez F. Putamen neurons process both sensory and motor information during a complex task. Brain Res. 2012;1466:70-81.

29. Romero MC, Bermudez MA, Vicente AF, Perez R, Gonzalez F. Activity of neurons in the caudate and putamen during a visuomotor task. Neuroreport. 2008;19(11):1141-1145.

30. Brovelli A, Nazarian B, Meunier M, Boussaoud D. Differential roles of caudate nucleus and putamen during instrumental learning. Neuroimage. 2011;57(4):1580-1590.

31. Ell SW, Marchant NL, Ivry RB. Focal putamen lesions impair learning in rule-based, but not information-integration categorization tasks. Neuropsychologia. 2006;44(10):1737-1751.

32. Laquitaine S, Piron C, Abellanas D, et al. Complex population response of dorsal putamen neurons predicts the ability to learn. PLoS One. 2013; 8(11):e80683.

33. Buchsbaum MS, Shihabuddin L, Brickman AM, et al. Caudate and putamen volumes in good and poor outcome patients with schizophrenia. Schizophr Res. 2003;64(1):53-62.

34. Tomycz ND, Friedlander RM. The experience of pain and the putamen: a new link found with functional MRI and diffusion tensor imaging. Neurosurgery. 2011;69(4):N12-N13.

35. Shin HY, Kang SY, Yang JH, et al. Use of the putamen/caudate volume ratio for early differentiation between parkinsonian variant of multiple system atrophy and Parkinson disease. J Clin Neurol. 2007;3(2):79-81.

36. Zhu Z, Gold BT, Chang CF, et al. Left middle temporal and inferior frontal regions contribute to speed of lexical decision: a TMS study. Brain Cogn. 2015;93:11-17.

37. Ilg UJ. The role of areas MT and MST in coding of visual motion underlying the execution of smooth pursuit. Vision Res. 2008;48(20): 2062-2069.

38. Ahlfors SP, Simpson GV, Dale AM, et al. Spatiotemporal activity of a cortical network for processing visual motion revealed by MEG and fMRI. J Neurophysiol. 1999;82(5):2545-2555.

39. Dunkley BT, Freeman TC, Muthukumaraswamy SD, Singh KD. Cortical oscillatory changes in human middle temporal cortex underlying smooth pursuit eye movements. Hum Brain Mapp. 2013;34(4):837-851.

40. Andersen RA, Essick GK, Siegel RM. Encoding of spatial location by posterior parietal neurons. Science. 1985;230(4724):456-458.

41. Caspers S, Eickhoff SB, Geyer S, et al. The human inferior parietal lobule in stereotaxic space. Brain Struct Funct. 2008;212(6):481-495.

42. Lewald J, Staedtgen M, Sparing R, Meister IG. Processing of auditory motion in inferior parietal lobule: evidence from transcranial magnetic stimulation. Neuropsychologia. 2011;49(2):209-215.

43. Huster RJ, Enriquez-Geppert S, Pantev C, Bruchmann M. Variations in midcingulate morphology are related to ERP indices of cognitive control. Brain Struct Funct. 2014;219(1):49-60.

44. Pereira MG, de Oliveira L, Erthal FS, et al. Emotion affects action: Midcingulate cortex as a pivotal node of interaction between negative emotion and motor signals. Cogn Affect Behav Neurosci. 2010;10(1):94-106.

45. Misra G, Coombes SA. Neuroimaging evidence of motor control and pain processing in the human midcingulate cortex. Cereb Cortex. 2015; 25(7):1906-1919.

46. Roebling R, Lerche $H$. Painful seizures associated with a lesion in the midcingulate cortex. J Neurol. 2009;256(6):1012-1014.

47. Uppal N, Wicinski B, Buxbaum JD, Heinsen H, Schmitz C, Hof PR. Neuropathology of the anterior midcingulate cortex in young children with autism. J Neuropathol Exp Neurol. 2014;73(9):891-902. 


\section{Publish your work in this journal}

Neuropsychiatric Disease and Treatment is an international, peerreviewed journal of clinical therapeutics and pharmacology focusing on concise rapid reporting of clinical or pre-clinical studies on a range of neuropsychiatric and neurological disorders. This journal is indexed on PubMed Central, the 'PsycINFO' database and CAS,

and is the official journal of The International Neuropsychiatric Association (INA). The manuscript management system is completely online and includes a very quick and fair peer-review system, which is all easy to use. Visit http://www.dovepress.com/testimonials.php to read real quotes from published authors.

Submit your manuscript here: http://www.dovepress.com/neuropsychiatric-disease-and-treatment-journal 